\title{
Quality of life and self-esteem in patients with psoriasis, vitiligo and healthy controls: A cross sectional study
}

\author{
Devanshi Sharma ${ }^{1, *}$, D M Mathur ${ }^{2}$, Jitendra Jeenger ${ }^{3}$, Manu Sharma ${ }^{4}$, Kalpana Gupta ${ }^{5}$ \\ ${ }^{\mathbf{1}}$ Post Graduate Resident, ${ }^{2,5}$ Professor and Head, ${ }^{3}$ Professor, ${ }^{4}$ Assistant Professor, ${ }^{1-4}$ Dept. of Psychiatry, ${ }^{5}$ Dept. of Dermatology \& \\ Venereology, Geetanjali Medical College \& Hospital, Udaipur, Rajasthan, India
}

*Corresponding Author: Devanshi Sharma

Email: devanshi2222@gmail.com

\begin{abstract}
Introduction: Psoriasis and vitiligo are among the most common chronic skin diseases, which have a negative impact on the psychosocial well-being. Patients may experience social rejection and stigmatization. So, the aim of this present study is to assess and compare the selfesteem and quality of life (QoL) in patients with vitiligo, psoriasis and healthy controls and its relationship with age and gender.

Materials and Methods: A cross-sectional and comparative study was conducted at a tertiary care hospital, Udaipur, Rajasthan, with a sample of total 150 cases, 50 each of psoriasis, vitiligo and healthy controls, aged between 18-64years. The assessment of all subjects was done for quality of life and self-esteem by using scales Short Form-36 (SF-36) and Rosenberg Self-Esteem Scale (RSES) respectively.

Results: The results of our study showed that the self-esteem\& quality of life was low in psoriatic patients when compared to vitiligo patients (p-0.000) and healthy controls (p-0.009). No relationship was found in patients of psoriasis and vitiligo between age and gender with regards to self-esteem and quality of life except poor social function domain of QoL, which was observed in middle age group (40-64years) patients of psoriasis.

Conclusion: While evaluating and arranging treatment of psoriasis and vitiligo patients, these results in poor psychosocial functioning should be considered and along with dermatologic interventions, psychiatric approaches should also be included for a better outcome.
\end{abstract}

Keywords: Quality of life, Self-esteem, Psoriasis, Vitiligo.

\section{Introduction}

Due to its responsiveness to emotional stimuli, skin has a special position in psychiatry. Its role is crucial in the development of self-esteem and ego integrity. ${ }^{1}$ Based on the fact that both being originated from the same ectodermal structure, the relationship between skin and brain is clear. ${ }^{2}$ So, psychodermatology makes up a common area of interest based on this mutual relationship between psychiatry and dermatology. ${ }^{3}$ Dermatology inpatients have been reported to have a higher prevalence of psychiatric disorders compared with general medical inpatients. ${ }^{4}$

Vitiligo is a depigmenting disorder. The total population affected with vitiligo is $0.5 \%$ to $1 \%$. Psoriasis is a psychocutaneous disease of the skin. It affects approximately $1.4-2 \%$ of the world's population, with men and women being affected equally. ${ }^{5}$ Psoriasis waxes and wanes in the form of episodes. ${ }^{6}$

Various degrees of psychological stress and altered Quality of Life (QoL) is experienced by the patients with vitiligo. ${ }^{7,8}$ Vitiligo patients also reported psychosocial impairments and altered QoL. ${ }^{9-12}$ The social stigma attached to vitiligo is known to produce deep psychological trauma. ${ }^{13}$ The forms of discrimination that patients with psoriasis experience includes the social rejection and alienation. ${ }^{14,15}$ The self-image of the patients is impaired by visible disfigurement. ${ }^{16-19}$

Previous studies have shown that, regardless of psoriasis severity, nearly $60 \%$ of the patients have a major effect on their QoL. ${ }^{20}$ Many patients with psoriasis suffer from social isolation and stigmatization. ${ }^{21,22}$ Furthermore, patients with severe psoriasis were found to have significant impaired work productivity. ${ }^{23,24}$ Psychological distress can lead to significant depression and social isolation. ${ }^{25}$

There is paucity of published psychosocial research in vitiligo and psoriasis available from India; most of the studies were targeted over psychiatric comorbidity in chronic disorders. Hence, the present study has been undertaken with the aim to study and compare the self-esteem and quality of life in patients with vitiligo and psoriasis and healthy controls and its relationship with age and gender.

\section{Materials and Methods}

This cross-sectional and comparative study was conducted at Geetanjali Medical College \& Hospital, Udaipur in the department of Psychiatry along with the department of Dermatology. Before starting the study, approval of the institutional ethics committee was taken. The study was conducted from August 2016-April 2017. One fifty, subjects who gave the informed consent were included in the present study. Out of which, 50 consecutive patients belonging to vitiligo and 50 consecutive patients belonging to psoriasis were taken. Age and gender matched 50 healthy controls preferably relatives and friends of the patients were taken. All patients were first examined by the consultant dermatologist who confirmed the diagnosis of psoriasis and vitiligo. In the study, patients of age between 18-64 years were included. Patients with past history of psychiatric disorders, those with serious/terminal medical illness, with other chronic dermatologic illness and with major medical or surgical illness were excluded. The investigator collected the socio-demographic and clinical variables on the proforma designed for the present study. Short Form-36 (SF-36) and 
Rosenberg Self-Esteem Scale (RSES) were used to assess QoL and self-esteem respectively in all subjects.

Short Form-36 (SF-36): This questionnaire consisted of 36 items forming 8 domains or scales: physical functioning; role physical; role emotional; bodily pain; social functioning; mental health; vitality; and general health perceptions. All questions are scored on a scale of 0 to 100 , with 100 representing the highest level of functioning possible.

Rosenberg Self-Esteem Scale (RSES): The RSES is a unidimensional instrument elaborated from a phenomenological conception of self-esteem that captures subjects' global perception of their own worth by means of a 10 -item scale. A four-point scale ranging from strongly agree to strongly disagree is used to answer these 10 items. The scale ranges from $0-30$, with 30 indicating the highest score possible. Scores between 15 and 25 are within normal range; scores below 15 suggest low self-esteem.

\section{Statistical Analysis}

Suitable statistical analysis (One Way ANOVA, Student ' $t$ ' test and Karl Pearson Coefficient of Correlation) using SPSS 20 software were subjected to the information so gained and data so collected and conclusions were drawn.

\section{Results}

Results of the study have been depicted below-
Table 1 shows the age \& gender profile of all three groups. The significantly high difference was observed in the self-esteem of psoriatic patients when compared to healthy controls (p- 0.009) and vitiligo patients (p- 0.000).And there was no difference in self-esteem of vitiligo patients when compared to healthy controls (p- 0.266) (Table 2). Psoriatic patients have significantly poor QoL when compared total score to healthy controls (p- 0.000$) \&$ in domains of physical health, emotional problems, emotional well-being \& social functioning with all having a p-value of 0.000 (Table 3). No significant difference was found in total scores of QoL \& its domains in vitiligo patients with healthy controls (p- 0.068 , $0.320,0.320,0.638)$. But the significant difference in Emotional well-being domain of QoL (Table 3). The differences in total scores and its domains of QoL in psoriatic patients and vitiligo patients was statistically highly significant (p-0.000) (Table 3). With regard to age (p-0.130, 0.138 ) (Table 4) and gender (p- 0.178, 0.809) (Table 4), no significant difference was found in self-esteem of psoriatic patients and vitiligo patients. The significant difference in total scores of QoL and its domain social functioning in psoriatic patients with regard to age were found and no difference in its other domains QoL with regard to age was observed. But no significant difference was found in total scores of QoL \& its domains in vitiligo patients with regard to age (Table 5). When compared gender and QoL with its domains in psoriatic patients and vitiligo patients no significant difference was observed (Table 6).

Table 1: Age and gender profile of psoriatic patients, vitiligo patients \& healthy controls-

\begin{tabular}{|c|c|c|c|c|}
\hline & Group & Psoriasis & Vitiligo & Healthy controls \\
\hline \multirow{2}{*}{ Age (Yrs) } & $18-39$ & $26(52.0 \%)$ & $29(58.0 \%)$ & $25(50.0 \%)$ \\
\cline { 2 - 5 } & $40-64$ & $24(48.0 \%)$ & $21(42.0 \%)$ & $25(50.0 \%)$ \\
\hline \multirow{2}{*}{ Sex } & Male & $37(74.0 \%)$ & $31(62.0 \%)$ & $29(58.0 \%)$ \\
\cline { 2 - 5 } & Female & $13(26.0 \%)$ & $19(38.0 \%)$ & $21(42.0 \%)$ \\
\hline
\end{tabular}

Table 2: Comparison of self-esteem (RSES) in psoriatic patients \& vitiligo patients \& healthy controls-

\begin{tabular}{|c|c|c|c|c|c|c|c|c|}
\hline & Type & $\mathbf{N}$ & Mean & $\begin{array}{c}\text { Std. } \\
\text { Deviation }\end{array}$ & $\begin{array}{c}\text { Std. Error } \\
\text { Mean }\end{array}$ & Mean Diff & 't' & P value \\
\hline \multirow{3}{*}{$\begin{array}{c}\text { RSES } \\
\text { (Rosenberg } \\
\text { Self-Esteem } \\
\text { Scale) }\end{array}$} & \multirow{3}{*}{$\begin{array}{c}\text { Psoriasis Vs } \\
\text { Healthy Controls }\end{array}$} & 50 & 16.6200 & 1.65233 & .23368 & 0.82000 & 2.664 & 0.009 \\
\hline & & 50 & 17.4400 & 1.41652 & .20033 & 0.30000 & 1.119 & 0.266 \\
\hline & & & & & & 1.12000 & 3.813 & 0.000 \\
\hline \multirow{2}{*}{ RSES } & \multirow{2}{*}{$\begin{array}{c}\text { Vitiligo Vs } \\
\text { Healthy Controls }\end{array}$} & 50 & 17.7400 & 1.25860 & .17799 & \multirow[t]{2}{*}{0.30000} & \multirow[t]{2}{*}{1.119} & \multirow[t]{2}{*}{0.266} \\
\hline & & 50 & 17.4400 & 1.41652 & .20033 & & & \\
\hline \multirow{2}{*}{ RSES } & \multirow{2}{*}{$\begin{array}{c}\text { Psoriasis Vs } \\
\text { Vitiligo }\end{array}$} & 50 & 16.6200 & 1.65233 & .23368 & \multirow[t]{2}{*}{1.12000} & \multirow[t]{2}{*}{3.813} & \multirow[t]{2}{*}{0.000} \\
\hline & & 50 & 17.7400 & 1.25860 & .17799 & & & \\
\hline
\end{tabular}


Table 3: Comparison of quality of life (sf-36) between psoriatic patients, vitiligo patients \& healthy controls-

\begin{tabular}{|c|c|c|c|c|c|c|c|c|}
\hline $\begin{array}{c}\text { SF-36 } \\
\text { (Short Form-36) }\end{array}$ & Type & $\mathbf{N}$ & Mean & $\begin{array}{c}\text { Std. } \\
\text { Deviation }\end{array}$ & $\begin{array}{c}\text { Std. Error } \\
\text { Mean }\end{array}$ & Mean Diff & 't' & P value \\
\hline \multirow{2}{*}{ Total } & Psoriasis & 50 & 69.27 & 25.035 & 3.541 & \multirow[t]{2}{*}{26.741} & \multirow[t]{2}{*}{7.452} & \multirow[t]{2}{*}{0.000} \\
\hline & Healthy controls & 50 & 96.01 & 4.122 & 0.583 & & & \\
\hline \multirow{2}{*}{$\begin{array}{l}\text { RLPH-Role } \\
\text { limitations due to } \\
\text { physical health }\end{array}$} & Psoriasis & 50 & 64.50 & 46.863 & 6.627 & \multirow[t]{2}{*}{35.500} & \multirow[t]{2}{*}{5.356} & \multirow[t]{2}{*}{0.000} \\
\hline & Healthy controls & 50 & 100.00 & 0.000 & 0.000 & & & \\
\hline \multirow{2}{*}{$\begin{array}{l}\text { RLEP- Role } \\
\text { limitations due to } \\
\text { emotional } \\
\text { problems }\end{array}$} & Psoriasis & 50 & 63.33 & 47.738 & 6.751 & \multirow[t]{2}{*}{36.667} & \multirow[t]{2}{*}{5.431} & \multirow[t]{2}{*}{0.000} \\
\hline & Healthy controls & 50 & 100.00 & 0.000 & 0.000 & & & \\
\hline \multirow{2}{*}{$\begin{array}{l}\text { EWB- Emotional } \\
\text { well-being }\end{array}$} & Psoriasis & 50 & 68.8800 & 20.89101 & 2.95443 & \multirow[t]{2}{*}{29.76000} & \multirow[t]{2}{*}{9.645} & \multirow[t]{2}{*}{0.000} \\
\hline & Healthy controls & 50 & 98.6400 & 6.29143 & .88974 & & & \\
\hline \multirow{2}{*}{$\begin{array}{l}\text { SF- Social } \\
\text { functioning }\end{array}$} & Psoriasis & 50 & 69.95 & 28.078 & 3.971 & \multirow[t]{2}{*}{24.910} & \multirow[t]{2}{*}{6.027} & \multirow[t]{2}{*}{0.000} \\
\hline & Healthy controls & 50 & 94.86 & 8.101 & 1.146 & & & \\
\hline \multicolumn{9}{|c|}{ Vitiligo Patients \& Healthy Controls- } \\
\hline \multirow{2}{*}{ Total } & \begin{tabular}{|c|} 
Vitiligo \\
\end{tabular} & 50 & 93.69 & 7.888 & 1.115 & \multirow[t]{2}{*}{2.321} & \multirow[t]{2}{*}{1.844} & \multirow[t]{2}{*}{0.068} \\
\hline & Healthy controls & 50 & 96.01 & 4.122 & 0.583 & & & \\
\hline \multirow{2}{*}{$\begin{array}{l}\text { RLPH-Role } \\
\text { limitations due to } \\
\text { physical health } \\
\end{array}$} & Vitiligo & 50 & 99.00 & 7.071 & 1.000 & \multirow[t]{2}{*}{1.000} & \multirow[t]{2}{*}{1.000} & 0.320 \\
\hline & Healthy Controls & 50 & 100.00 & 0.000 & 0.000 & & & \\
\hline RLEP- Role & Vitiligo & 50 & 98.67 & 9.429 & 1.333 & 1.333 & 1.000 & 0.320 \\
\hline $\begin{array}{l}\text { limitations due to } \\
\text { emotional } \\
\text { problems }\end{array}$ & Healthy controls & 50 & 100.00 & 0.000 & 0.000 & & & \\
\hline EWB- Emotional & Vitiligo & 50 & 89.3600 & 12.71631 & 1.79836 & 9.28000 & 4.625 & 0.000 \\
\hline well-being & Healthy controls & 50 & 98.6400 & 6.29143 & .88974 & & & \\
\hline SF- Social & Vitiligo & 50 & 95.75 & 10.598 & 1.499 & 0.890 & 0.472 & 0.638 \\
\hline functioning & Healthy controls & 50 & 94.86 & 8.101 & 1.146 & & & \\
\hline Psoriatic Patients & \& Vitiligo Patients & & & & & & & \\
\hline Total & \begin{tabular}{|l|} 
Psoriasis \\
\end{tabular} & 50 & 69.27 & 25.035 & 3.541 & 24.420 & 6.578 & 0.000 \\
\hline 1otal & Vitiligo & 50 & 93.69 & 7.888 & 1.115 & & & \\
\hline RLPH-Role & Psoriasis & 50 & 64.50 & 46.863 & 6.627 & 34.500 & 5.147 & 0.000 \\
\hline $\begin{array}{l}\text { limitations due to } \\
\text { physical health }\end{array}$ & Vitiligo & 50 & 99.00 & 7.071 & 1.000 & & & \\
\hline RLEP- Role & Psoriasis & 50 & 63.33 & 47.738 & 6.751 & 35.334 & 5.135 & 0.000 \\
\hline $\begin{array}{l}\text { limitations due to } \\
\text { emotional } \\
\text { problems }\end{array}$ & Vitiligo & 50 & 98.67 & 9.429 & 1.333 & & & \\
\hline EWB- Emotional & Psoriasis & 50 & 68.8800 & 20.89101 & 2.95443 & 20.48000 & 5.921 & 0.000 \\
\hline well-being & Vitiligo & 50 & 89.3600 & 12.71631 & 1.79836 & & & \\
\hline SF- Social & Psoriasis & 50 & 69.95 & 28.078 & 3.971 & 25.800 & 6.079 & 0.000 \\
\hline functioning & Vitiligo & 50 & 95.75 & 10.598 & 1.499 & & & \\
\hline
\end{tabular}

Table 4: Relationship of self-esteem (RSES) with regard to age \& gender in psoriatic patients and vitiligo patients-

\begin{tabular}{|c|c|c|c|c|c|c|c|c|}
\hline $\begin{array}{c}\text { RSES } \\
\text { (Rosenberg Self- } \\
\text { Esteem Scale) }\end{array}$ & $\begin{array}{c}\text { Age \& } \\
\text { Gender }\end{array}$ & $\mathbf{N}$ & Mean & Std. Deviation & Std. Error Mean & Mean Difference & 't' & P value \\
\hline \multirow[b]{2}{*}{ Psoriasis } & \multirow{2}{*}{$\begin{array}{c}18-39 \text { vs } \\
40-64\end{array}$} & 26 & 16.9615 & 1.50946 & .29603 & \multirow[t]{2}{*}{0.71154} & \multirow[t]{2}{*}{1.543} & \multirow[t]{2}{*}{0.130} \\
\hline & & 24 & 16.2500 & 1.75078 & .35738 & & & \\
\hline \multirow[b]{2}{*}{ Vitiligo } & \multirow{2}{*}{$\begin{array}{c}18-39 \text { vs } \\
40-64\end{array}$} & 29 & 17.9655 & 1.40109 & .26018 & \multirow[t]{2}{*}{0.53695} & \multirow[t]{2}{*}{1.508} & \multirow[t]{2}{*}{0.138} \\
\hline & & 21 & 17.4286 & 0.97834 & .21349 & & & \\
\hline \multirow[b]{2}{*}{ Psoriasis } & \multirow{2}{*}{$\begin{array}{l}\text { Male vs } \\
\text { Female }\end{array}$} & 37 & 16.4324 & 1.59061 & .26149 & \multirow[t]{2}{*}{0.72141} & \multirow[t]{2}{*}{1.366} & \multirow[t]{2}{*}{0.178} \\
\hline & & 13 & 17.1538 & 1.77229 & .49155 & & & \\
\hline \multirow[b]{2}{*}{ Vitiligo } & \multirow{2}{*}{$\begin{array}{l}\text { Male vs } \\
\text { Female }\end{array}$} & 31 & 17.7742 & 1.28348 & 0.23052 & \multirow[t]{2}{*}{0.08998} & \multirow[t]{2}{*}{0.243} & \multirow[t]{2}{*}{0.809} \\
\hline & & 19 & 17.6842 & 1.24956 & 0.28667 & & & \\
\hline
\end{tabular}


Table 5: Relationship of quality of life with (sf-36) with regard to age in psoriatic patients and vitiligo patients

\begin{tabular}{|c|c|c|c|c|c|c|c|c|c|}
\hline \multirow{11}{*}{ Psoriasis } & $\begin{array}{c}\text { SF-36 } \\
\text { (Short Form-36) }\end{array}$ & Age & $\mathbf{N}$ & Mean & $\begin{array}{c}\text { Std. } \\
\text { Deviation }\end{array}$ & $\begin{array}{l}\text { Std. Error } \\
\text { Mean }\end{array}$ & $\begin{array}{c}\text { Mean } \\
\text { Difference }\end{array}$ & 't' & P value \\
\hline & \multirow{2}{*}{ Total } & $18-39$ & 26 & 76.32 & 24.963 & 4.896 & \multirow[t]{2}{*}{14.686} & \multirow[t]{2}{*}{2.147} & \multirow[t]{2}{*}{0.037} \\
\hline & & $40-64$ & 24 & 61.64 & 23.257 & 4.747 & & & \\
\hline & \multirow{2}{*}{$\begin{array}{c}\text { RLPH-Role } \\
\text { limitations due to } \\
\text { physical health }\end{array}$} & $18-39$ & 26 & 75.00 & 43.012 & 8.435 & \multirow[t]{2}{*}{21.875} & \multirow[t]{2}{*}{1.679} & \multirow[t]{2}{*}{0.100} \\
\hline & & $40-64$ & 24 & 53.13 & 49.074 & 10.017 & & & \\
\hline & \multirow{2}{*}{$\begin{array}{l}\text { RLEP- Role } \\
\text { limitations due to } \\
\text { emotional } \\
\text { problems }\end{array}$} & $18-39$ & 26 & 73.08 & 45.234 & 8.871 & \multirow[t]{2}{*}{20.301} & \multirow[t]{2}{*}{1.522} & \multirow[t]{2}{*}{0.134} \\
\hline & & $40-64$ & 24 & 52.78 & 49.066 & 10.016 & & & \\
\hline & \multirow{2}{*}{$\begin{array}{c}\text { EWB- Emotional } \\
\text { well-being }\end{array}$} & $18-39$ & 26 & 73.0769 & 20.75557 & 4.07050 & \multirow[t]{2}{*}{8.74359} & \multirow[t]{2}{*}{1.497} & \multirow[t]{2}{*}{0.141} \\
\hline & & $40-64$ & 24 & 64.3333 & 20.49532 & 4.18359 & & & \\
\hline & \multirow{2}{*}{$\begin{array}{c}\text { SF- Social } \\
\text { functioning }\end{array}$} & 18-39 & 26 & 78.75 & 28.074 & 5.506 & \multirow[t]{2}{*}{18.333} & \multirow[t]{2}{*}{2.418} & \multirow[t]{2}{*}{0.019} \\
\hline & & $40-64$ & 24 & 60.42 & 25.310 & 5.166 & & & \\
\hline \multirow{10}{*}{ Vitiligo } & \multirow{2}{*}{ Total } & 18-39 & 29 & 95.08 & 4.376 & 0.813 & \multirow[t]{2}{*}{3.315} & \multirow[t]{2}{*}{1.485} & \multirow[t]{2}{*}{0.144} \\
\hline & & $40-64$ & 21 & 91.77 & 10.905 & 2.380 & & & \\
\hline & \multirow{2}{*}{$\begin{array}{l}\text { RLPH-Role } \\
\text { limitations due to } \\
\text { physical health }\end{array}$} & $18-39$ & 29 & 100.00 & 0.000 & 0.000 & \multirow[t]{2}{*}{2.381} & \multirow[t]{2}{*}{1.180} & \multirow[t]{2}{*}{0.244} \\
\hline & & $40-64$ & 21 & 97.62 & 10.911 & 2.381 & & & \\
\hline & \multirow{2}{*}{$\begin{array}{l}\text { RLEP- Role } \\
\text { limitations due to } \\
\text { emotional } \\
\text { problems }\end{array}$} & $18-39$ & 29 & 100.00 & 0.000 & 0.000 & \multirow[t]{2}{*}{3.175} & \multirow[t]{2}{*}{1.180} & \multirow[t]{2}{*}{0.244} \\
\hline & & $40-64$ & 21 & 96.83 & 14.549 & 3.175 & & & \\
\hline & EWB- Emotional & $18-39$ & 29 & 89.9310 & 11.39527 & 2.11605 & 1.35961 & 0.370 & 0.713 \\
\hline & well-being & $40-64$ & 21 & 88.5714 & 14.60333 & 3.18671 & & & \\
\hline & SF- Social & $18-39$ & 29 & 97.41 & 7.748 & 1.439 & 3.961 & 1.314 & 0.195 \\
\hline & functioning & $40-64$ & 21 & 93.45 & 13.474 & 2.940 & & & \\
\hline
\end{tabular}

Table 6: Relationship of quality of life with (sf-36) with regard to gender in psoriatic patients and vitiligo patients -

\begin{tabular}{|c|c|c|c|c|c|c|c|c|c|}
\hline & $\begin{array}{c}\text { SF-36 } \\
\text { (Short Form-36) }\end{array}$ & Sex & $\mathbf{N}$ & Mean & $\begin{array}{c}\text { Std. } \\
\text { Deviation }\end{array}$ & $\begin{array}{l}\text { Std. Error } \\
\text { Mean }\end{array}$ & $\begin{array}{c}\text { Mean } \\
\text { Difference }\end{array}$ & 't' & $P$ value \\
\hline \multirow{10}{*}{ Psoriasis } & \multirow{2}{*}{ Total } & \multirow{2}{*}{$\begin{array}{l}\text { Male vs } \\
\text { Female }\end{array}$} & 37 & 66.25 & 24.556 & 4.037 & \multirow[t]{2}{*}{11.619} & \multirow[t]{2}{*}{1.456} & \multirow[t]{2}{*}{0.152} \\
\hline & & & 13 & 77.87 & 25.338 & 7.028 & & & \\
\hline & \multirow{2}{*}{$\begin{array}{l}\text { RLPH-Role limitations due to } \\
\text { physical health }\end{array}$} & \multirow{2}{*}{$\begin{array}{l}\text { Male vs } \\
\text { Female }\end{array}$} & 37 & 60.14 & 47.675 & 7.838 & \multirow[t]{2}{*}{16.788} & \multirow[t]{2}{*}{1.114} & \multirow[t]{2}{*}{0.271} \\
\hline & & & 13 & 76.92 & 43.853 & 12.163 & & & \\
\hline & \multirow{2}{*}{$\begin{array}{l}\text { RLEP- Role limitations due to } \\
\text { emotional problems }\end{array}$} & \multirow{2}{*}{$\begin{array}{l}\text { Male vs } \\
\text { Female }\end{array}$} & 37 & 58.56 & 48.690 & 8.005 & \multirow[t]{2}{*}{18.366} & \multirow[t]{2}{*}{1.199} & \multirow[t]{2}{*}{0.237} \\
\hline & & & 13 & 76.92 & 43.853 & 12.163 & & & \\
\hline & \multirow[t]{2}{*}{ EWB- Emotional well-being } & $\begin{array}{l}\text { Male vs } \\
\text { Female }\end{array}$ & 37 & 66.8108 & 20.93089 & 3.44102 & \multirow[t]{2}{*}{7.95842} & \multirow[t]{2}{*}{1.186} & \multirow[t]{2}{*}{0.241} \\
\hline & & & 13 & 74.7692 & 20.42121 & 5.66382 & & & \\
\hline & \multirow[t]{2}{*}{ SF- Social functioning } & $\begin{array}{l}\text { Male vs } \\
\text { Female }\end{array}$ & 37 & 67.50 & 27.329 & 4.493 & \multirow[t]{2}{*}{9.423} & \multirow[t]{2}{*}{1.042} & \multirow[t]{2}{*}{0.303} \\
\hline & & & 13 & 76.92 & 30.124 & 8.355 & & & \\
\hline \multirow{9}{*}{ Vitiligo } & \multirow[t]{2}{*}{ Total } & $\begin{array}{l}\text { Male vs } \\
\text { Female }\end{array}$ & 31 & 94.39 & 4.980 & 0.894 & \multirow[t]{2}{*}{1.846} & \multirow[t]{2}{*}{0.800} & \multirow[t]{2}{*}{0.428} \\
\hline & & & 19 & 92.55 & 11.216 & 2.573 & & & \\
\hline & \multirow{2}{*}{$\begin{array}{l}\text { RLPH-Role limitations due to } \\
\text { physical health }\end{array}$} & $\begin{array}{l}\text { Male vs } \\
\text { Female }\end{array}$ & 31 & 100.00 & 0.000 & 0.000 & \multirow[t]{2}{*}{2.632} & \multirow[t]{2}{*}{1.286} & \multirow[t]{2}{*}{0.205} \\
\hline & & & 19 & 97.37 & 11.471 & 2.632 & & & \\
\hline & \multirow{2}{*}{$\begin{array}{l}\text { RLEP-Role limitations due to } \\
\text { emotional problems }\end{array}$} & $\begin{array}{l}\text { Male vs } \\
\text { Female }\end{array}$ & 31 & 100.00 & 0.000 & 0.000 & 3.509 & 1.286 & 0.205 \\
\hline & & & 19 & 96.49 & 15.295 & 3.509 & & & \\
\hline & EWB- Emotional well-being & $\begin{array}{l}\text { Male vs } \\
\text { Female }\end{array}$ & 31 & 89.6774 & 11.35015 & 2.03855 & 0.83531 & 0.223 & 0.824 \\
\hline & & & 19 & 88.8421 & 15.00097 & 3.44146 & & & \\
\hline & SF- Social functioning & $\begin{array}{l}\text { Male vs } \\
\text { Female }\end{array}$ & 31 & 95.97 & 9.347 & 1.679 & 0.573 & 0.184 & 0.855 \\
\hline
\end{tabular}




\section{Discussion}

The present study was aimed to assess and compare QoL and self-esteem of patients diagnosed with psoriasis, vitiligo and healthy controls. Though many studies have been done to assess the QoL but on self-esteem very few studies are available.

Self-esteem can be an important part of individual's personality. Self-esteem (SE) describes the belief and confidence in one's own worth, strengths, efficacy and success. It is a subjective state of feeling formed by the individual's realistic evaluation of himself or herself. ${ }^{26}$ According to our results, psoriatic patients had low selfesteem on RSES but vitiligo patients had better self- esteem than did psoriasis patients. Too low self-esteem can leave people feeling defeated or depressed. On psychosocial wellbeing, psoriasis has a negative impact. Therefore, psoriasis may lead to a decrease in the self-esteem. ${ }^{27,28}$ Psoriasis is an illness that involves both the skin and joints, and thus may negatively affect self-esteem. ${ }^{29}$ In line with our findings, Ersin Aydin et al also observed patients with psoriasis have lower self-esteem than the control group. ${ }^{30}$ While our finding is contradictory with previous study, they concluded that both groups did not differ in overall self-esteem. ${ }^{31}$ Porter $\mathbf{J}$ et al showed that the patients with vitiligo who cope well with their disfigurement have higher self-esteem than a matched control group without the disorder and those who cope poorly have significantly lower self-esteem, which suggests that response to disfiguring diseases is affected by basic ego strength. ${ }^{32}$ In contrast to our findings, a previous study assessing self-esteem in 644 vitiligo patients reported reduced self-esteem among vitiligo patients. ${ }^{33}$

It maybe because vitiligo patients exhibited better adjustment in their daily routines because of its painless nature, while psoriatic patients reported more interpersonal problems in our study.

According to WHO, QoL is a broad ranging concept affected in a complex way by the person's physical health, psychological state, level of independence, social relationships, personal beliefs and their relationship to salient features of their environment. ${ }^{39}$ It is defined as individual's perception of their position in life in the context of the culture and value systems in which they live and in relation to their goals, expectations, standards and concerns. ${ }^{39}$ Psoriatic patients have significantly poor quality of life when compared total score to vitiligo patients ( $\mathrm{p}-0.000)$ and healthy controls (p-0.000) \& in domains of physical health, emotional problems, emotional well-being \& social functioning ( $\mathrm{p}$ 0.000 ) according to our study. Mahsa Ghajarzadeh et al also observed that psoriasis cases had more impairment in quality of life than alopecia or vitiligo cases as shown by higher mean DLQI (Dermatology Life Quality Index) scores among alopecia or vitiligo cases than psoriasis cases. ${ }^{28,34}$ Other researchers also observed that QoL impairment in psoriasis was greater than in vitiligo. ${ }^{35-37}$ It maybe because physical problems such as pain and itching were reported more by psoriasis patients more as compared to vitiligo patients in our study.
The impact of vitiligo on QoL is controversial. Lucy Beth N Sangam et al found that $95 \%$ of patients of vitiligo had elevated DLQI against $30 \%$ in control group $(P<$ 0.001). ${ }^{38}$ Linthorst Homan et al. assessed QoL in 245 multiracial adult patients $(\geq 18$ years) with generalized vitiligo. These vitiligo patients had a low mental Health Related Quality of Life (HRQL). ${ }^{40}$

We didn't find any statistically significant difference between vitiligo and healthy control with regard to QoL. It maybe because patients with vitiligo had good social occupational functioning in our study.

In our study, with regard to age and gender, no significant difference in self-esteem in psoriatic patients and vitiligo patients was observed. In a study by Bohm et al observed no gender differences $(\mathrm{P}<0.05)$ in the frequency of items related to appearance and socialization; however, men reported greater work-related stresses ${ }^{43}$ Middle age group psoriatic patients had poor social functioning according to our study. Maire Karelson et al observed that in vitiligo patients the mean DLQI was not associated with gender and age.$^{44}$ QoL score did not significantly vary among different types of disease, age and marital status groups observed by Soodabeh Zandi et al. ${ }^{41}$ In line with our findings, the same results were reported by Wong \& Baba in a survey of Malaysian patients with vitiligo. ${ }^{42}$ On the contrary, several studies have reported lower QoL in women with vitiligo, who may be more emotional and sensitive about their appearance ${ }^{44,45}$ It maybe because of the differences in social beliefs about vitiligo in this part of country.

\section{Conclusion}

The profound effect of psoriasis and vitiligo on the QoL and self-esteem of the patients is important to understand. The burden of psoriasis and vitiligo on the QoL and selfesteem is quantified by the present study. Vitiligo and psoriasis are chronic diseases and they are disappointing and distressing for patients. During the evaluation and treatment of psoriasis and vitiligo patients, it should be considered that these are not only dermatological diseases, but also reduce self-esteem and quality of life, therefore for a better outcome, along with dermatologic interventions, psychiatric approaches should also be included. Further research could also consider appropriate interventions targeted towards improving quality of life and self-esteem of the patients.

Strengths of the study is that different kind of groups are compared. Different domains of QoL were assessed. This is the first study of its kind which was conducted in the state of Rajasthan, where there is paucity of published psychosocial research in vitiligo and psoriasis.

There are some limitations to this study. The sample size being compared in the study was small. The study was done in a tertiary care center and the samples were selected consecutively, such a sample does not include many more sufferers treated elsewhere, hence not a true representative of the actual problem. 
Acknowledgment: Nil.

\section{Conflicts of Interest: Nil.}

\section{References}

1. Domonkos AN. Pruritus, Cutaneous neuroses and Neurocutaneous dermatoses: In Anrews GC, Domonkos AN (editors). Andrew's Disease of the skin: clinical dermatology. 6th ed. Philadelphia: WB Sounders Co., 1971, 61-76.

2. Koblenzer CS. Psychosomatic concepts in dermatology. Arch Dermatol. 1983;119:501-512.

3. Mercan S, Altunay Kıvaç İ. Psychodermatology: a collaboration between psychiatry and dermatology. Turk $J$ Psychiatry. 2006;17:305-313.

4. Jafferany M, Vander Stoep A, Dumitrescu A, Hornung RL. The knowledge, awareness, and practice patterns of dermatologists toward psychocutaneous disorders: Results of a survey study. Int J Dermatol. 2010;49:784-9.

5. Bhosle MJ, Kulkarni A, Feldman SR, Balkrishnan R. Quality of life in patients with psoriasis. Health Qual Life Outcomes. 2006;4:35.

6. Campalani E, Barker JNWN. The clinical genetics of psoriasis. Curr Genomics. 2005;6(1):51-60.

7. Kim do Y, Lee JW, Whang SH, Park YK, Hann SK, Shin YJ. Quality of life for Korean patients with vitiligo: Skindex-29 and its correlation with clinical profiles. $J$ Dermatol. 2009;36:317-322.

8. Parsad D, Dogra S, Kanwar AJ. Quality of life in patients with vitiligo. Health Qual Life Outcomes. 2003;1:58.

9. Kent G, Al'Abadie M. Psychologic effects of vitiligo: a critical incident analysis. J Am Acad Dermatol. 1996;35:895-898.

10. Sampogna F, Raskovic D, Guerra L, Pedicelli C, Tabolli S, Leoni $\mathrm{L}$, et al. Identification of categories at risk for high quality of life impairment in patients with vitiligo. $\mathrm{Br} \mathrm{J}$ Dermatol. 2008;159:351-359.

11. Porter JR, Beuf AH, Lerner AB, Nordlund JJ. The effect of vitiligo on sexual relationships. J Am Acad Dermatol. 1990;22:221-222.

12. Belhadjali H, Amri M, Mecheri A, Doarika A, Khorchani H, Youssef M, et al. Vitiligo and quality of life: a case-control study. Ann Dermatol Venereol. 2007;134:233-236.

13. Sharma N, Koranne RV, Singh RK. Psychiatric morbidity in psoriasis and vitiligo: a comparative study. $J$ Dermatol. 2001;28:419-423.

14. Hrehorów E, Salomon J, Matusiak L. Patients with psoriasis feel stigmatized. Acta Derm Venereol. 2012;92(1):67-72.

15. Kimball $\mathrm{AB}$, Jacobson $\mathrm{C}$, Weiss $\mathrm{S}$. The psychosocial burden of psoriasis. Am J Clin Dermatol. 2005;6(6):383-392.

16. Vardy D, Besser A, Amir M. Experiences of stigmatization play a role in mediating the impact of disease severity on quality of life in psoriasis patients. $B r J$ Dermatol. 2002;147(4):736-742.

17. Schmid-Ott G. Future trends in psychodermatological psoriasis research: somatopsychic or psychosomatic focus? Dermatol Psychosom. 2003;4(3):129-130.

18. Schmid-Ott G, Schallmayer S, Calliess IT. Quality of life in patients with psoriasis and psoriasis arthritis with a special focus on stigmatization experience. Clin Dermatol. 2007;25(6):547-554.

19. Schmid-Ott G, Künsebeck HW, Jäger B, et al. Significance of the stigmatization experience of psoriasis patients: a 1-year follow-up of the illness and its psychosocial consequences in men and women. Acta Derm Venereol. 2005;85(1):27-32.

20. Stern RS, Nijsten T, Feldman SR, Margolis DJ, Rolstad T (2004) Psoriasis is common, carries a substantial burden even when not extensive, and is associated with widespread treatment dissatisfaction. The journal of investigative dermatology Symposium proceedings/the Society for Investigative Dermatology, Inc [and] European Society for Dermatological Research. 2004;9:136-139.

21. Gupta MA, Gupta AK, Watteel GN (1998) Perceived deprivation of social touch in psoriasis is associated with greater psychologic morbidity: an index of the stigma experience in dermatologic disorders. Cutis 61: 339-342.

22. Schmid-Ott G, Jaeger B, Kuensebeck HW, Ott R, Lamprecht F (1996) Dimensions of stigmatization in patients with psoriasis in a "Questionnaire on Experience with Skin Complaints". Dermatol. 1996;193:304-310.

23. Horn EJ, Fox KM, Patel V, Chiou CF, Dann F. Association of patient-reported psoriasis severity with income and employment. J Am Acad Dermatol. 2007;57:963-971.

24. Pearce DJ, Singh S, Balkrishnan R, Kulkarni A, Fleischer $\mathrm{AB}$. The negative impact of psoriasis on the workplace. $J$ Dermatolog Treat. 2006;17:24-28.

25. National Institute of Arthritis and Musculoskeletal and Skin Diseases, US Department of Health \& Human Services, 2003. Healthtopics: psoriasis, http://www.niams. nih.gov /hi/topics/psoriasis/ psoriafs.htm.

26. Ho J, Lee A, Kaminsky L, Wirrell E. Self-concept, attitude toward illness and family functioning in adolescents with type 1 diabetes. Paediatr Child Health. 2008;13:600-604.

27. Cupertino Felipe, Niemeyer-Corbellini Jo ao Paulo, RamoseSilva Marcia, Psychosomatic Aspects of Vitiligo, Clinics in Dermatology (2017),

28. Mahsa Ghajarzadeh, Maryam Ghiasi, and Shahrbanoo Kheirkhah, Associations between Skin Diseases and Quality of Life: A Comparison of Psoriasis, Vitiligo, and Alopecia Areata, May 2012

29. Dewing KA. Management of patients with psoriatic arthritis. Nurse Pract. 2015;40:40-46.

30. Aydin E, Atis G, Bolu A, Aydin C, Karabacak E, Dogan B, Ates MA. Identification of anger and self-esteem in psoriasis patients in a consultation-liaison psychiatry setting: a case control study. Klinik Psikofarmakoloji Bulteni. 2016;26(3):3.

31. Judith R. Porter. Psychosocial effect of vitiligo: A comparison of vitiligo patients with "normal" control subjects, with psoriasis patients, and with patients with other pigmentary disorders, Journal of American Academy of Dermatology, August 1986

32. Porter J, Beuf AH, Nordlund JJ, Lerner AB. Psychological reaction to chronic skin disorders: a study of patients with vitiligo. General hospital psychiatry. 1979;1(1):73-77.

33. Kent G, Al'Abadie M. Factors affecting responses on Dermatology Life Quality Index items among vitiligo sufferers. Clin Exp Dermatol. 1996;21:330-303.

34. Amir Mufaddel, Abdelghani Elsheikh Abdelgani, Psychiatric Comorbidity in Patients with Psoriasis, Vitiligo, Acne, Eczema and Group of Patients with Miscellaneous Dermatological Diagnoses, J Psychiatry. 2014, 4, 168-175

35. Benchikh H, Abarji H, Nani S. Quality of life is more affected in psoriasis than vitiligo: a study of 40 Moroccan patients. Dermatol Open J. 2016;1(1): 9-13.

36. Hanan M. Saleh, Samar Abdallah M. Salem, Rania S ElSheshetawy and Afaf M. Abd El-Samei, Comparative Study of Psychiatric Morbidity and Quality of Life in Psoriasis, Vitiligo and Alopecia Areata. Egypt Dermatol Online J. 2008;4(1):2.

37. K. Ongenae, N. Van Geel, S. De Schepper and J.M. Naeyaert, Department of Dermatology, Ghent University Hospital, Belgium, Effect of vitiligo on self-reported health-related quality of life, March 2004.

38. Sangma LN, Nath J. and Bhagabati D, Quality of Life and Psychological Morbidity in Vitiligo Patients: A Study in a Teaching Hospital from North-East India. Indian J Dermatol. 2015;60(2):142-146. 
39. Gholami A, Jahromi LM, Zarei E, Dehghan A. Application of WHOQOL-BREF in measuring quality of life in health-care staff. Int J Prev Med. 2013;4(7):809.

40. Homan MW, Spuls PI, de Korte J, Bos JD, Sprangers MA, van der Veen JW. The burden of vitiligo: Patient characteristics associated with quality of life. J Am Acad Dermatol. 2009;61:411-420.

41. Zandi S, Farajzadeh S, Saberi N, Effect of vitiligo on selfreported quality of life in southern part of Iran. J Pak Assoc Dermatol. 2016;21(1):4-9.

42. Wong SM, Baba R. Quality of life among Malaysian patients with vitiligo. Int J Dermatol. 2012;51(2):158-161.

43. Böhm D, Stock Gissendanner S, Bangemann K, Snitjer I, Werfel T, Weyergraf A, Schulz W, Jäger B, Schmid-Ott G.
Perceived relationships between severity of psoriasis symptoms, gender, stigmatization and quality of life. $J$ Eur Acad Dermatol Venereol. 2013;27(2):220-206.

44. Karelson M, Silm H and Kingo K, Quality of Life and Emotional State in Vitiligo in an Estonian Sample: Comparison with Psoriasis and Healthy Controls, Acta Dermato-venereologica. 2013; 93(4): 446-50.

45. Borimnejad L, Yekta ZP, Nikbakht-Nasrabadi A, Firooz A. Quality of life with vitiligo: Comparison of male and female Muslim patients in Iran. Gend Med. 2006;3:124-130. 